\title{
Nucleotide Bond
}

National Cancer Institute

\section{Source}

National Cancer Institute. Nucleotide Bond. NCI Thesaurus. Code C157223.

The phosphodiester bonds that are formed between nucleotides in a nucleic acid. 